\title{
The K-BTE medical laser device's special circuit
}

\section{Introduction}

The KBTE Device is created in a proprietary method where we assemble in a special circuitry $120 \mathrm{~V}$ - DC/AC/RC-reverse current (canceling the magnetic from the electric) which then is transferred through water. After deleting the magnetic aspect we extract the bio electrons from this water (electric fluid), controlling their direction and strength (Micro and Nano amperes frequency). Lastly we add in extracted electrons enriched with a number of natural acids. We incorporate these natural acids into our Biological Agents Device (Figure 1). Today's scientific formula for electromagnetic current power is: $\mathrm{I}=\mathrm{V}: \mathrm{R}(\mathrm{I}=$ current; $\mathrm{V}=$ Voltages and $\mathrm{R}=$ Human resistance to ground in Ohms). Today's standard technological advanced medical surgeries use lasers which can only perform at the strength/frequency of Mili Amperes, which is: $1 \mathrm{~A}=1,000$ Mili Amperes. Please understand that if the human body's resistance to ground is $1,000 \mathrm{Ohms}$ and using 120 Voltages which is 1.2Mili Amperes or 1,200Micro Amperes, then it's threshold impact, would result in electric shock and death. This is today's current official technological development in laser medicine.

Our KBTE Device's maximum strength output among the first energetic field and the second energetic field are 2 Direct Current DC producing Micro Amperes, the maximum level is $15 \mathrm{DC}$ Micro Amperes, with an average of 8.5 Direct Current, DC. This measured current is confirmed for the body's resistance from 1,000
Volume 2 Issue 3 - 2017

\begin{abstract}
Nick Kostovi
Inventor of laser medical device K-BTE and Therapist of Bio Electric Surgery and Therapy, USA

Correspondence: Nick Kostovic, Inventor of laser medical device K-BTE and Therapist of

Bio Electric Surgery and Therapy, 3838 Carson Street, \# Suite 307, Torrance, CA 90503, USA, Fax + I-540-6946, Tel + I-310543-0309, + I-310-381-9105, Email nick@kbhealthcenter.com
\end{abstract}

Received: February 21, 2017| Published: March 02, 2017

to 100,000 Ohms or more, it doesn't matter dry or wet. The severity of any lethal shock is absolutely eliminated as these voltages cannot produce a dangerous shock. The technician's hands feel a slight tingling sensation. The client's hands on the first energetic field feels a slight repelling action (not an attracting sensation), because we have separated electro from magnetic. The KBTE device is such an innovative discovery because of this special circuitry of DC/direct current/AC/alternating current/and RC/reverse current (Figure $2 \& 3$ ).

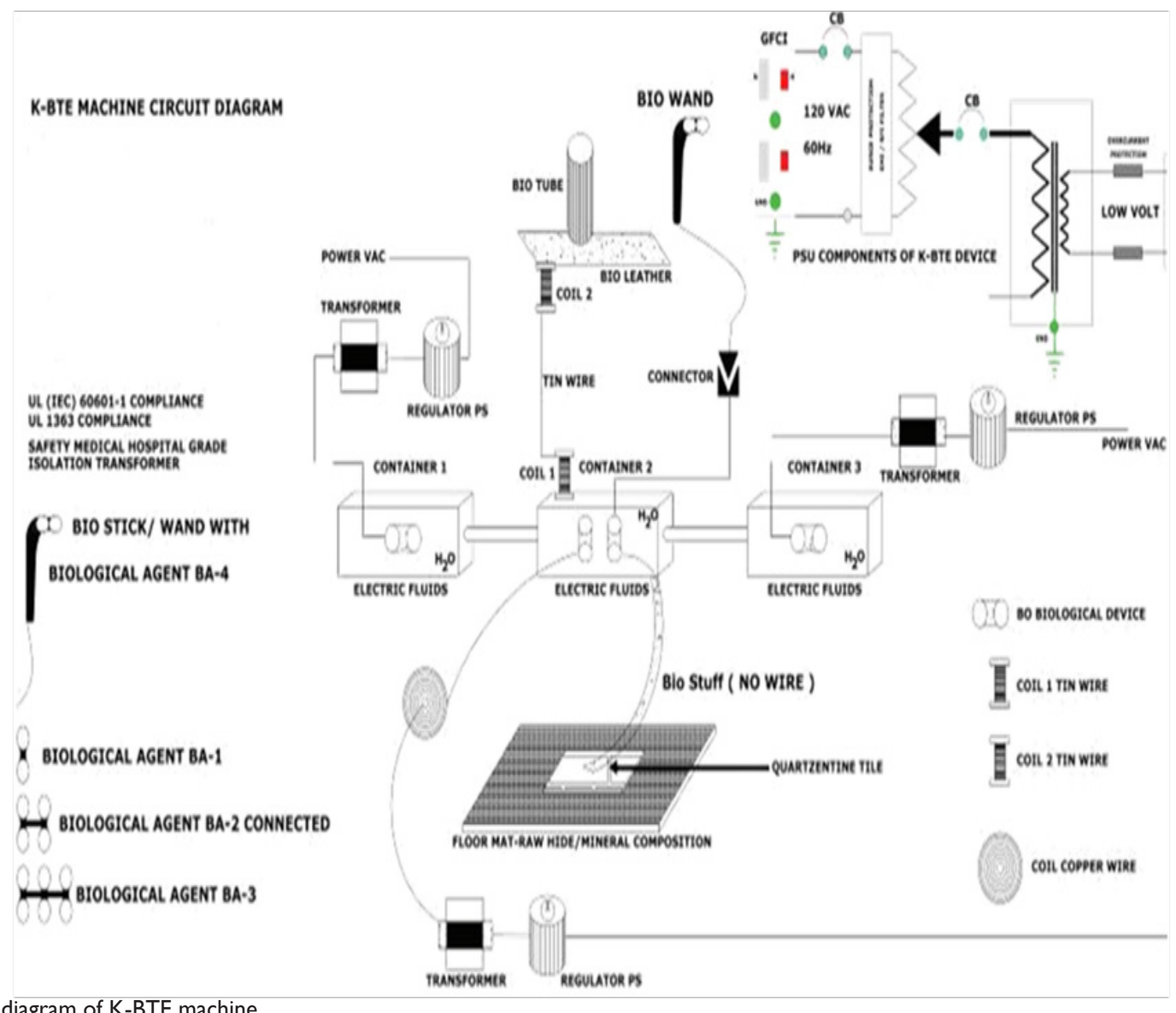

Figure I Circuit diagram of K-BTE machine. 


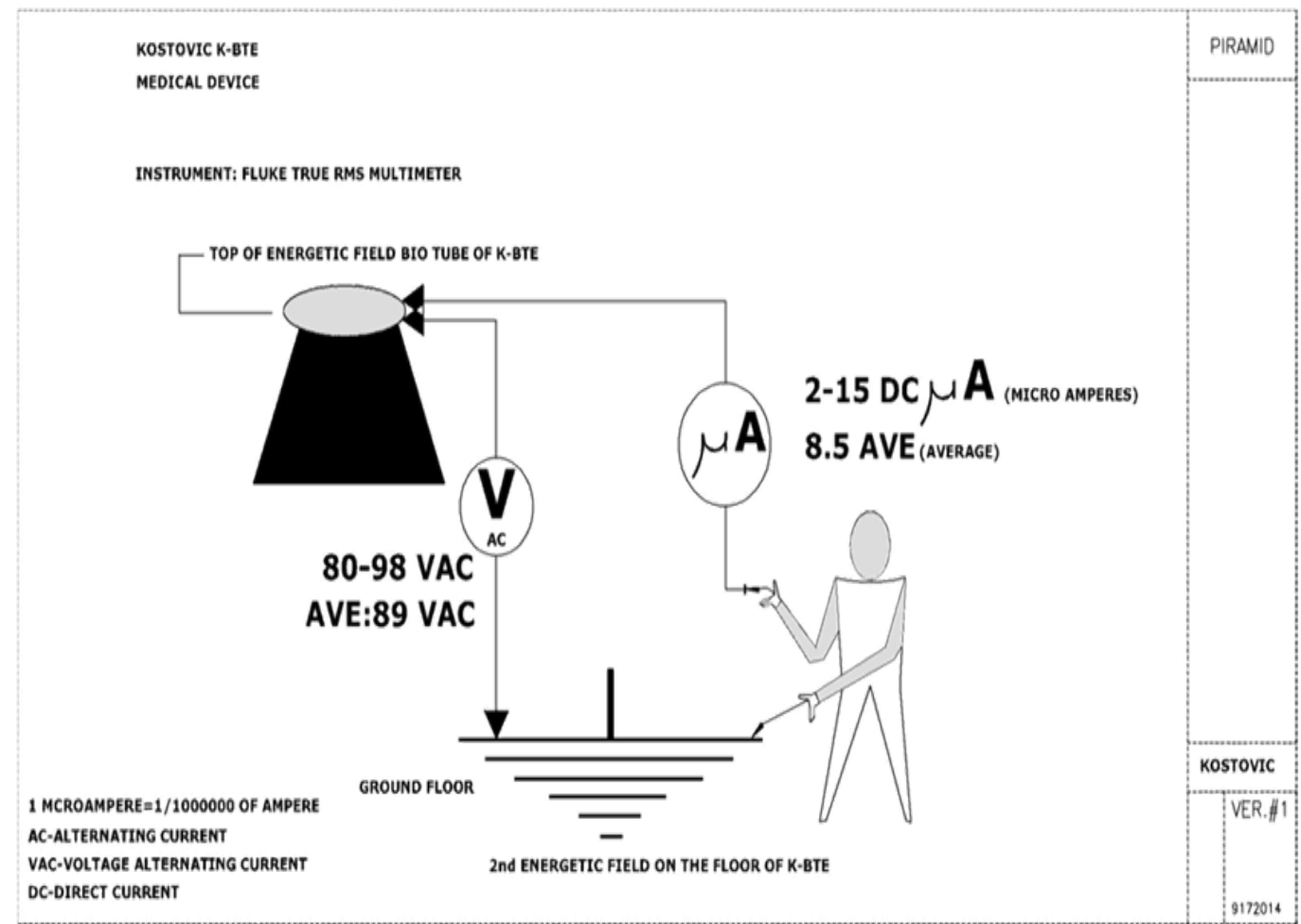

Figure 2 Clinic data K-BTE medical device.

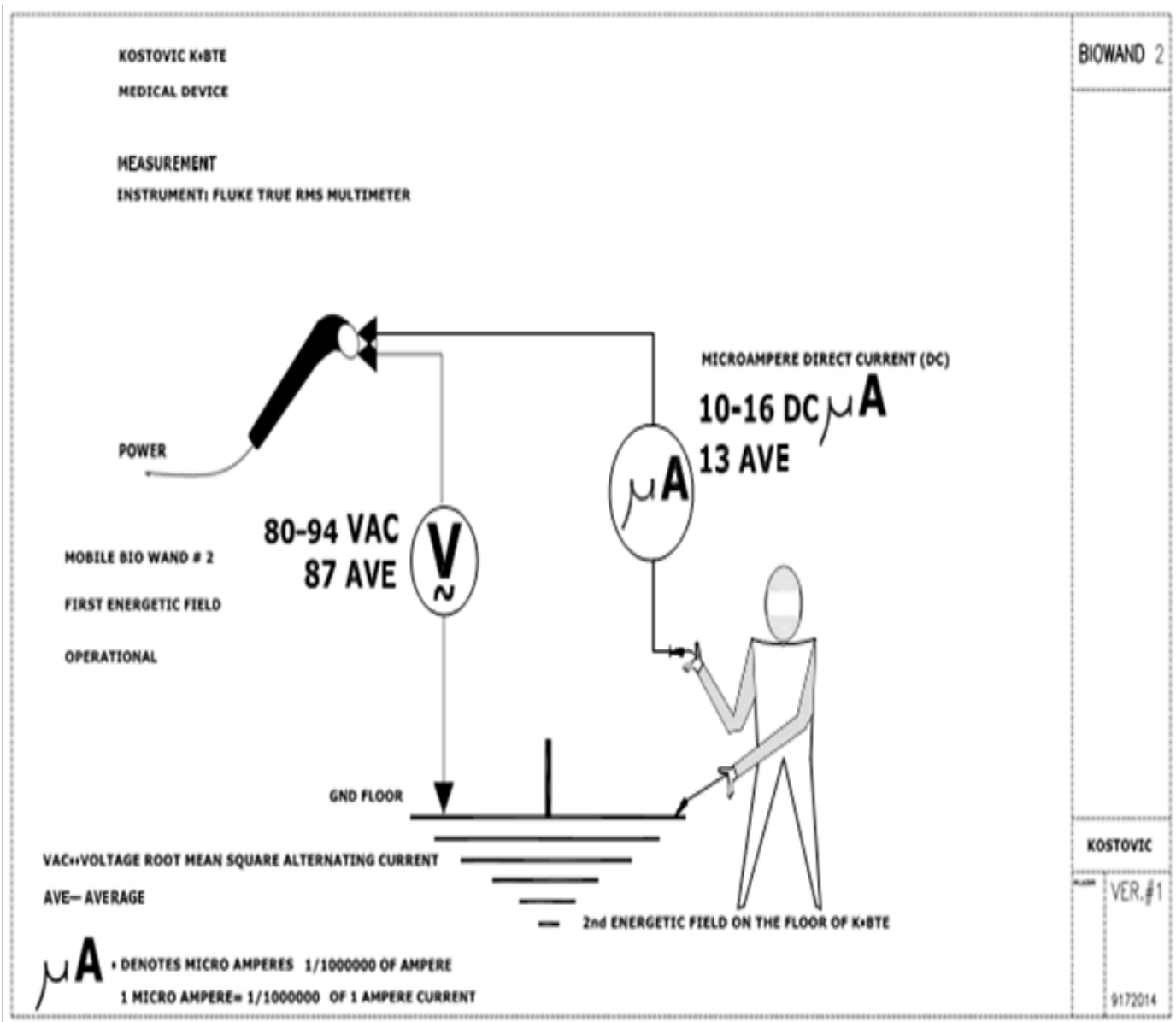

Figure 3 Clinic data Bio wand \# 2. 
Knowing the Human body's resistance to ground could be 1,000 Ohms (wet) yet using 120 voltages of AC we very safely produce the gentle effect of 12-15Micro Amperes (DC). By utilizing this newly discovered Reverse Current and separating electro from magnetic we prevent electromagnetic shock. Our K-BTE device utilizing these gentle Micro Amperes frequencies we can effectively burn and disperse oxidized proteins. This frequency is 10,000 times less than the strength of today's developed laser technology. These energetic acupuncture type penetrations on the surface of the skin are soft, not at all an electric shock. It doesn't matter the human body's resistance is 1,000 , or 100,000 or more it just doesn't matter anymore because we are producing such a low frequency of Micro Amperes. We can even bring it down further to the Nano Amperes frequency which is 1 billionth of the amplitude. K-BTE releases sheaves of the elementary laser's beams of the biological electricity consisted from enriched bio electron's, photons through biological and mineral agents such as gold, silver and pyrite-Fe2. The biological electricity converts acids from virgin olive oil, fig, and lemon, etc. as liquid ("values of different energetic conductors") in energetic form of enriched bio electron's photons. The Virgin olive oil contains natural acids such as Oleic and Leno oleic, antioxidants, not saturated fats, polyphenol. These ingredients are very important in the protection of healthy cells from destructible free radicals.

\section{Evidences related to the effectiveness of use of laser medical device K-BTE in malignant cancer cases:}

Knowing the Human body's resistance to ground could be $1,000 \mathrm{Ohms}$ (wet) yet using 120 voltages of AC we very safely produce the gentle effect of 2-15Micro Amperes (DC). In the brain case we always started releasing of bio electron's photons with speed/ strength by 10 ( 0.01 Micro Amperes) to 1,000Nano Amperes (1 Micro Amperes), and later by 1 to 10 Micro Amperes. The laser device K-BTE releases the elementary laser's beams of bio electron's photons which are able to successfully attack and eradicate most types of malignant cancer with metastases including prostate, breast, lungs, pancreatic, liver, brain, etc. The healthy cells are simultaneously strengthened and as noted, without side effects. For brain cancer, treatments begin with striking the malignant brain tumor by bio electron's photons enriched with natural acids, in the form of energetic acupuncture or elementary laser's beams of the biological electricity, penetrating deep throughout the brain. The areas with lesions and formations were reduced or completely eliminated with no harm to healthy brain nerve cells, or any other healthy cells (Figure $4 \& 5$ ).

On a daily basis our cells are always being born and dying. When we are young our bodies can disperse and get rid of all the dead cells. We have cells that eat these dead oxidized proteins. But with aging the dead cells are produced faster than the aging body can get rid of. When the oxygen and protein rich blood supply is prevented from interacting with the muscle fibers due to this build up of dead cells. These nourishing proteins become oxidized proteins. These oxidized proteins become our number 1 enemy, as they attract parasites, microbes and bacteria's etc. So now the human body not only has dying fiber tissue, the building blocks of muscle tissue but we also have diseases developing. If we are not able to clean this declining fiber tissue from the dead cells and oxidized proteins, then the tissue will die, creating a domino like effect killing more and more fiber tissues, the building blocks of the human body's organs.

For the first time in history our discovery enables us to maintain the physical organs. We do this by first cleansing the fiber tissues, burning away the dead cells and oxidized proteins with our wireless acupuncture therapy. After we have burned away the dead cells and oxidized proteins making space for the healthy blood supply to finally reach the fiber tissues, then body can rebuild healthy fiber tissue. Recently we have been registered a new non-profit organization "Kostovic Bio-Electric Laser Institute", and we would like to expose our technology to spread and open centers worldwide. This technology is the most efficient therapy method in the world and we are proud to state it (Figure 6-8).

\section{K-BTE BIOLOGICAL agents devices}

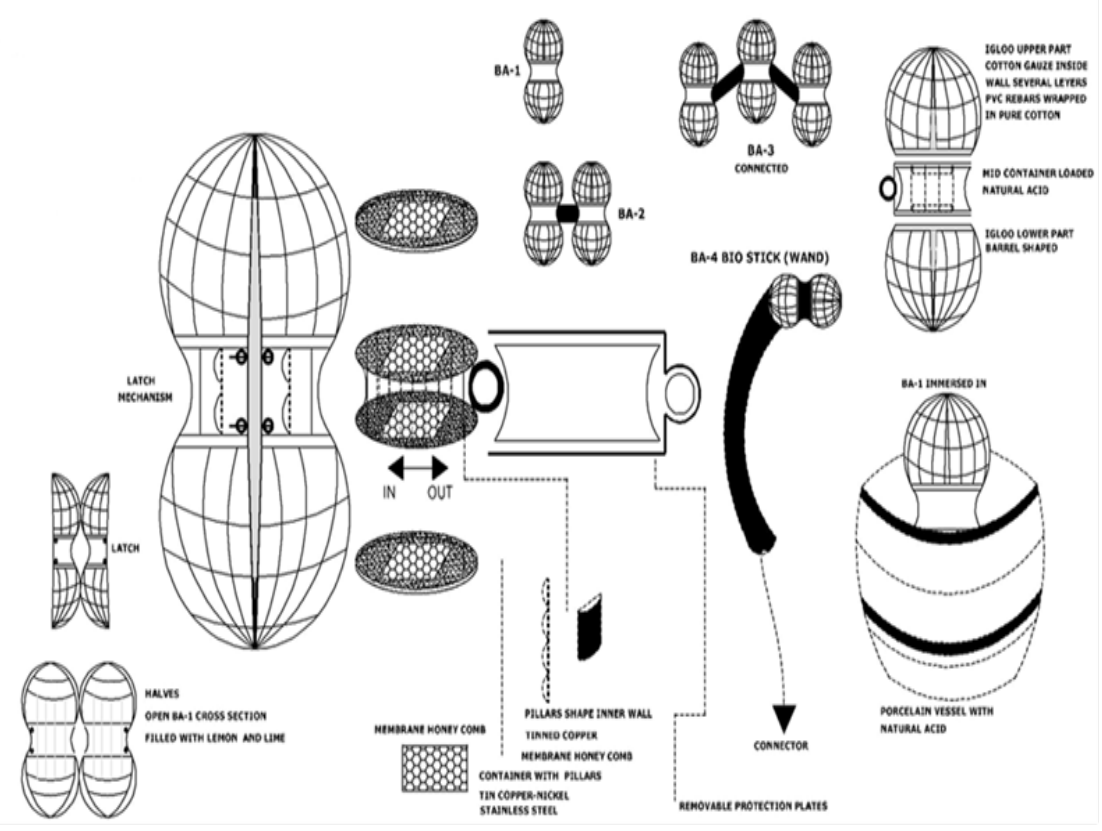

Figure 4 K-BTE biological agents $20 \mathrm{I}$ model. 
K-BTE BIOLOGICAL agents devices

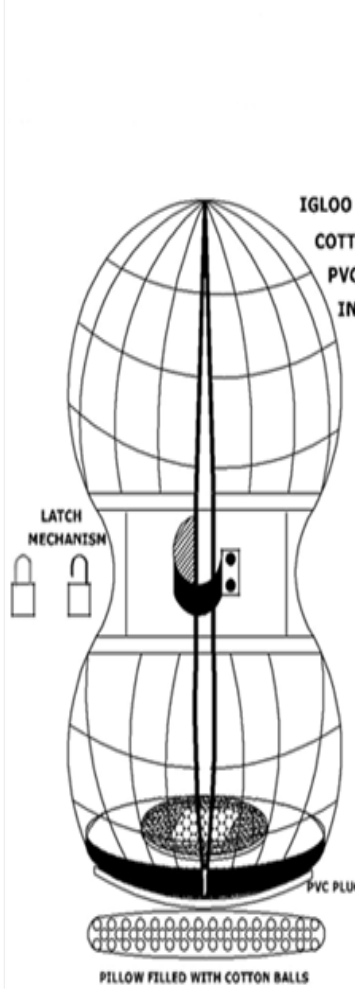

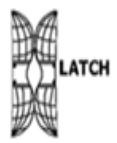

GLOO BA UPPER PART

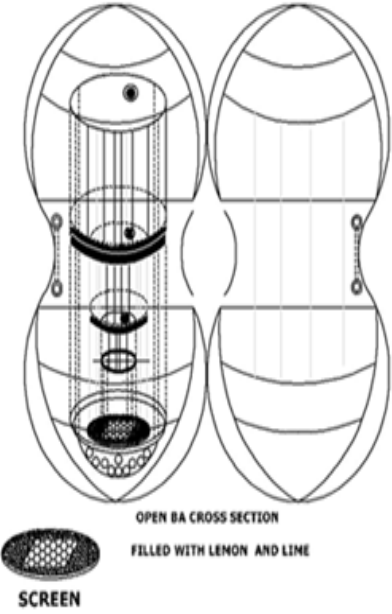

BARS

SOLID TINNED COPPER WELDED INSIDE
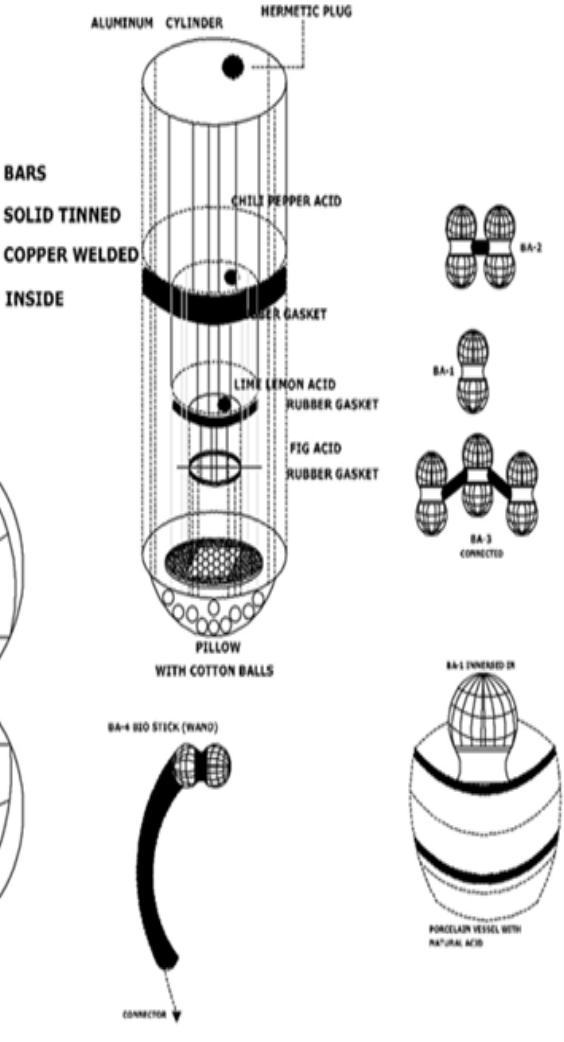

Figure 5 K-BTE biological agents 202 model.
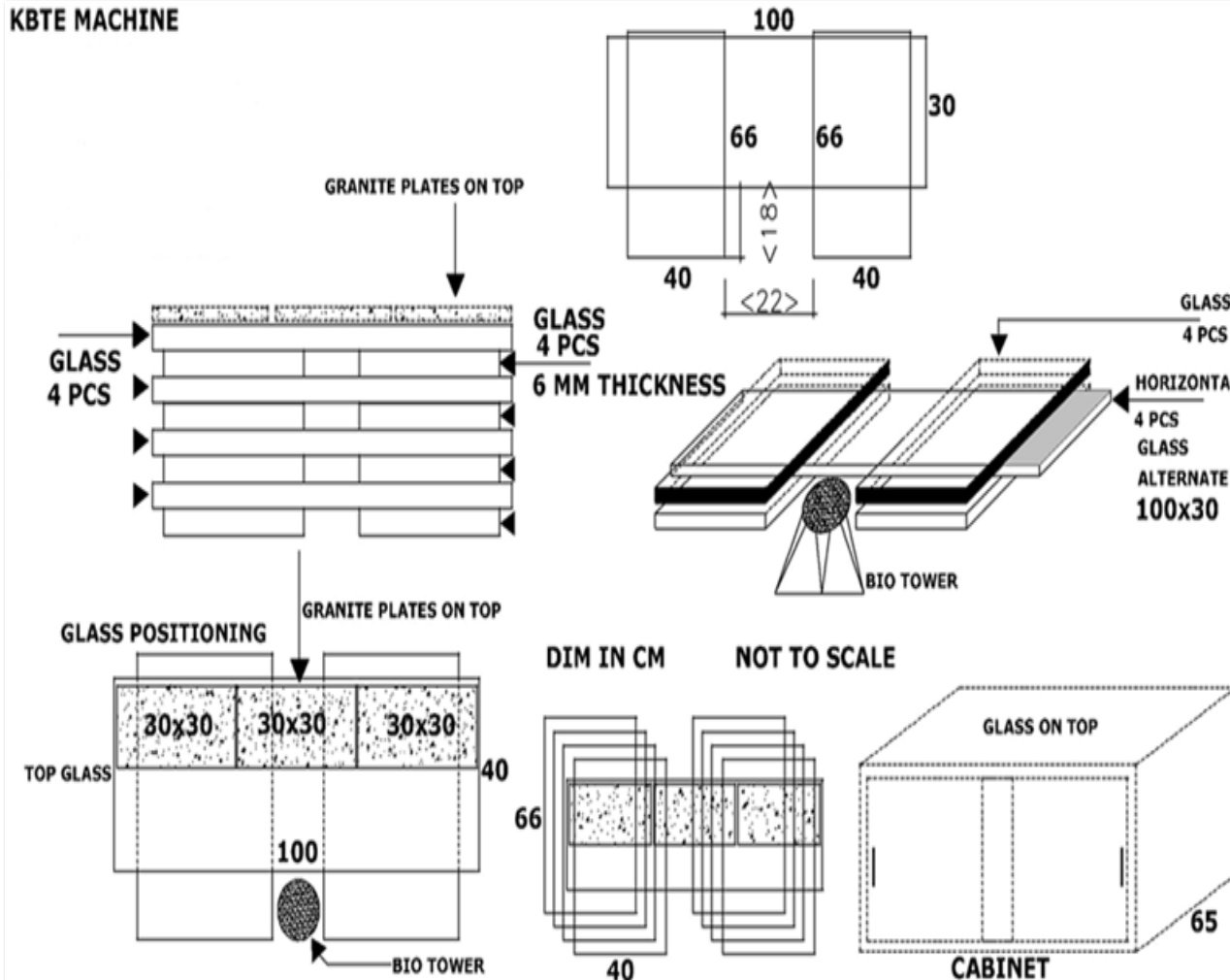

DIM IN CM NOT TO SCALE
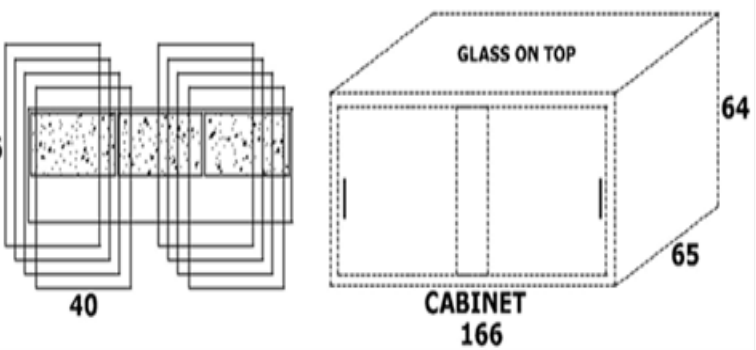

Figure 6 K-BTE machine (glassNiksa I I model). 


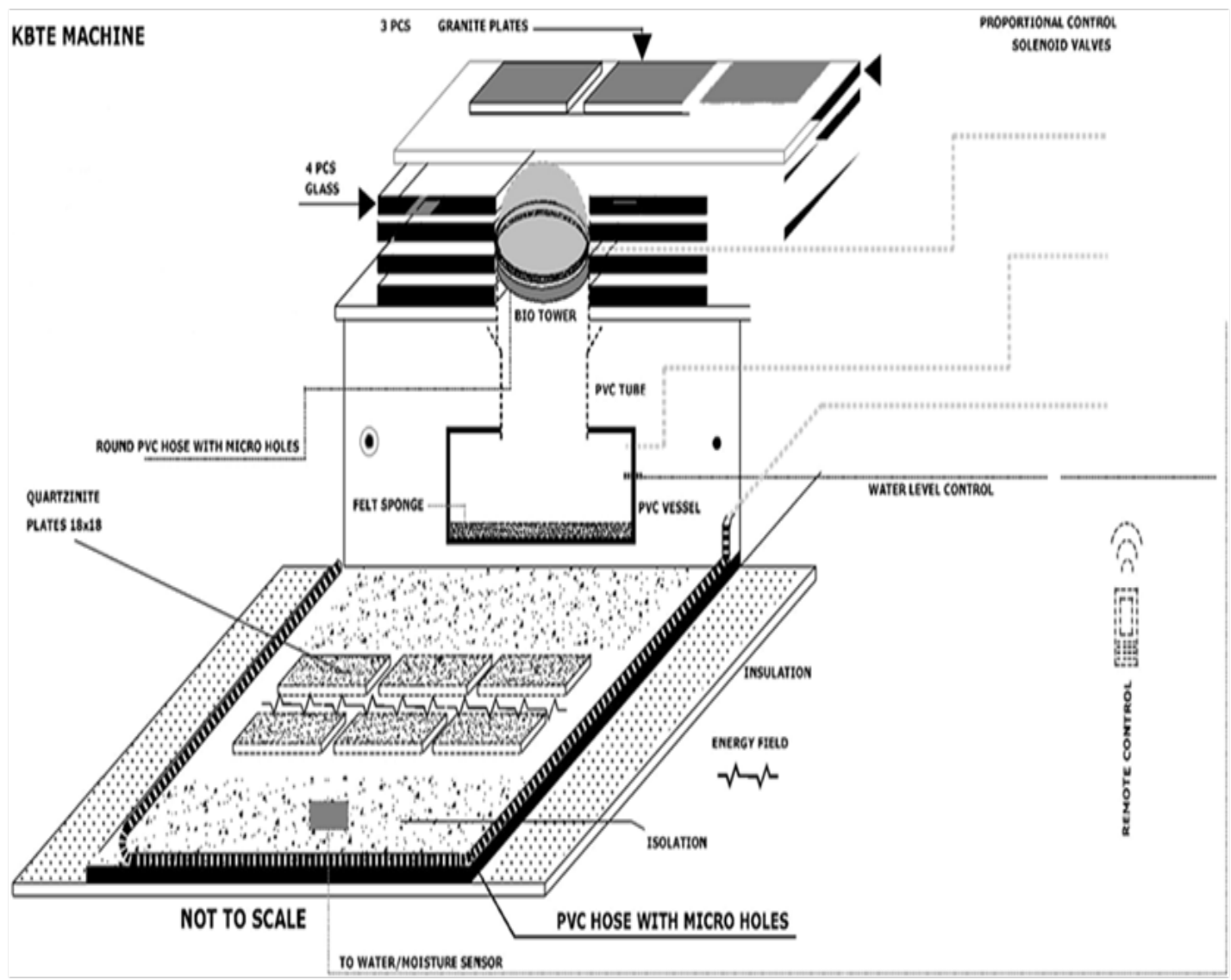

Figure 7 K-BTE machine (Solenoidswater model).

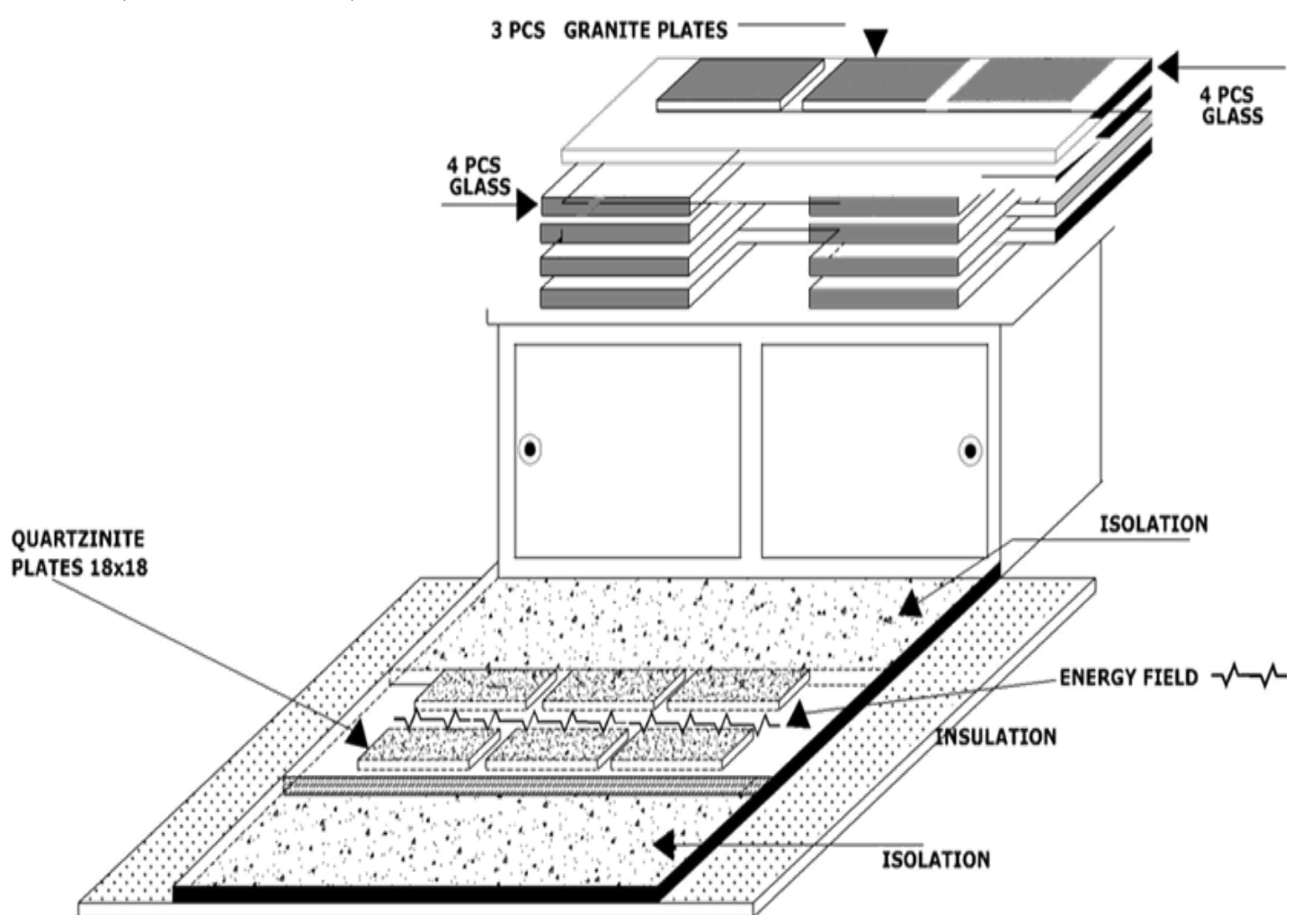

Figure 8 K-BTE machine (Niksaall model). 


\section{Acknowledgements}

None.

\section{Conflict of interest}

The author declares no conflict of interest. 